\title{
A Model-based Parameter Optimization Control Strategy for Trajectory Tracking with Torque and Velocity Constraints
}

\section{Min Luo (D 36205007@qq.com )}

University of Electronic Science and Technology of China https://orcid.org/0000-0002-3154-1657

\section{Xiaorong Hou}

University of Electronic Science and Technology of China

\section{Xiaoxue Li}

University of Electronic Science and Technology of China

Jinbo Lu

Southwest Petroleum University

Jing Yang

University of Electronic Science and Technology of China

\section{Research Article}

Keywords: Wheeled robots, Trajectory tracking, Parameter optimization, Velocity constraints, Torque constraints

Posted Date: November 5th, 2021

DOl: https://doi.org/10.21203/rs.3.rs-975228/v1

License: (c) (i) This work is licensed under a Creative Commons Attribution 4.0 International License. Read Full License 


\title{
A model-based parameter optimization control strategy for trajectory tracking with torque and velocity constraints
}

\author{
Min Luo · Xiaorong Hou · Xiaoxue Li · Jinbo Lu • Jing Yang
}

Received: date / Accepted: date

\begin{abstract}
The wheeled robots trajectory tracking control methods rarely constrain the torque and speed at the same time. In actual application, the torque and speed of the robot cannot exceed the saturation limit of the actuator. This paper develops a model-based trajectory tracking parameter optimization controller with both velocity and torque constraints, using a gradient descent parameter iterative learning strategy to minimize the settling time index of the system. Trajectory tracking time optimization methods usually require a given analytical expression of the system time, while this time optimization method only requires that the settling time is solvable. The MATLAB simulation experiments show that the proposed parameter optimization controller for trajectory tracking can perform velocity and torque constraints while having a relatively good overall rapidity time index. If the resolution of the robot sensor can meet the design requirements, the optimization method can strictly control the system torque maximum to a reasonably small expected value. When the resolution of the robot sensor is limited, this optimization method can restrict the system torque maximum within a reasonable saturation constraint range.
\end{abstract}

Keywords Wheeled robots · Trajectory tracking · Parameter optimization - Velocity constraints · Torque constraints

M. Luo $\cdot$ X. Hou $\left({ }^{凶}\right) \cdot$ X. Li $\cdot$ J. Yang

School of Automation Engineering, University of Electronic Science and Technology of China, Chengdu 611731, China

E-mail: houxr@uestc.edu.cn

M. Luo · J. Lu

School of Electrical Engineering and Information, Southwest

Petroleum University, Chengdu 610500, China

M. Luo

E-mail: 36205007@qq.com

\section{Introduction}

The robot trajectory tracking control has become a hot field of robot research [1]-[6]. Scientists have studied various wheeled robots controller design methods [7][10]. When designing the trajectory tracking controller, the saturation constraint of the actuator must be considered [11]-[22]. In the existing research on trajectory tracking control, there are many papers on the velocity constraint control of the actuator [11]-[16], and a few papers on the torque constraint control [17]-[18]. Both constraints are rarely considered at the same time [19].

\subsection{Robot trajectory tracking with velocity constraints}

In practice, the operating technical specifications of motors are always limited. High angular or linear velocity may lead to the robot losing stability and rollover or exceeding the safe travel range to cause a collision or accident. Therefore, it is necessary to control the speed limit of the robot [11]-[16].

$\mathrm{Yu}$ et al. [11] used the position error vector to construct the Lyapunov function and designed a trajectory tracking control law for a nonholonomic unicycle vehicle to realize the position vector errorless tracking with speed limitations. The simulation illustrated that tuning the controller parameters can realize limiting the velocity constraints.

Liu et al. [12] designed a controller with velocity constraints allowing the unicycle formation to track the reference trajectory, and the steady-state error converges to zero. The leader and follower of the system are both subjected to linear and angular velocity constraints due to the physical limitation. The experiment results proved 
that the velocity commands would not exceed the physical restraints.

Yang et al. [13] employed a model predictive control to a nonholonomic wheeled mobile robot for trajectory tracking in the presence of velocity constraints and other softening constraints. The presented control strategy contained a feedforward controller and a feedback controller to achieve trajectory tracking smoothly and reach the requirements of the softening constraints. For improving real-time robustness, a linearised tracking error model was utilized to predict system behaviors.

Guo et al. [14] took the linear and angular velocity limits into account to design a unicycle-type robot trajectory tracking controller. The tracking experiments illustrated that the proposed controller has strong robustness and stability. The controller tracked a variety of different trajectories accurately.

$\mathrm{Xu}$ et al. [15] presented a tracking control strategy integrating a biologically inspired backstepping controller and a torque controller with an unscented Kalman filter and Kalman filter to avoid and reduce the velocity jumps and overshoots and provide smooth velocity commands. The upper and lower bounds of the speed are set by the $\mathrm{B}$ and $\mathrm{D}$ parameters in the biologically inspired neural network, and the control constraints are realized. The proposed method can track the desired trajectory more accurately, provide smooth velocity commands and reduce the effects of the noises.

Chen et al. [16] devised a model-based controller and an adaptive neural network controller for a wheeled mobile robot with velocity constraints. This paper has accomplished two control law devisals, stability analysis, simulation, and practical experiments. The model controller will track the required trajectory well and limit the speed to meet the saturation constraint. The model-based control method enables the position error to converge to a small neighborhood of zero without violating the speed constraint. And the adaptive neural network is suitable for uncertain robot systems to approximate uncertain or time-varying parameters and improve the control robustness.

\subsection{Robot trajectory tracking with torque constraints}

The torque is physically bounded by the practical power supply limitation. Excessive torque causes saturation of the actuator, and it may deteriorate the tracking performance of the controller, especially in transient response. A long-term torque saturation may cause thermal, electrical, or mechanical failure of the actuator. Accordingly, controlling the torque to make the actu- ator not saturated has attracted the attention of the robot field [17]-[18].

Ailon et al. [17] addressed the trajectory tracking control of differentially driven nonholonomic wheeled mobile robots subjecting to bounded torque inputs. In this article, the applied torques are bounded for a known constant. Under the constraint conditions, the controller can ensure accurate tracking and guarantee that the vehicle does not deviate from the trajectory. The presented control algorithm applied hyperbolic functions for tracking a reference path with an associated timing law under the applied forces and torques constraints.

Wang et al. [18] proposed a model predictive control and Extended State Observer-based Adaptive Sliding Mode Control (ESO-AMSC) for formation control of Mecanum-Wheeled Mobile Robots (MWMR) with physical constraints and model uncertainties. The designed adaptive sliding mode controller with extended state observer has torque saturation constraints. The simulation results show that the linear model predictive controller can deal with the kinematic constraints of MWMRs, and the ESO-ASMC can address the input torque saturation constraints and remove the effect of model uncertainties and unknown disturbances.

\subsection{Main contributions}

As mentioned above, the wheeled robot should restrict the torque and speed. These two constraints need to be considered at one time to make the designed robot control system operate safely [19].

Yue et al. [19] designed a robust model predictive control strategy to handle the trajectory tracking for an underactuated two-wheeled inverted pendulum vehicle with various physical constraints. The authors treat the tilt angle, angular velocity, longitudinal and rotational travel speeds, and input torques as the physical constraints from outside the system. And they employed a Lyapunov candidate to handle the physical limitations from outside. Simultaneously, they exploited the model predictive control to solve the diversity of the physical constraints and unavoidable disturbances on the inner-loop system. The numerical simulations verified that the controller could track the designated trajectory with various physical limits and interferences.

Compared with the control method proposed in [11][15], [17], [19], which can not achieve the error-free tracking well or the settling time is too long, the model-based control method presented in [16] can quickly realize the error-free track of the position vector, and the control method is simple. The controller designed in [18] only carried out a comparative discussion of several different 
control methods and did not provide the experimental curve diagram and data of the position vector tracking of the reference trajectory.

This paper develops a model-based parameter optimization scheme for the speed and torque constraints. The general time optimization method needs an analytical time expression [20]-[28]. The model-based parameter optimal method proposed in this paper only needs the settling time to be calculatable. Here we take the gradient descent optimal method to design the modelbased parameter optimal strategy for settling time [29][31]. With the proposed method, the position and velocity errors can converge to zero. The main contributions of this paper are as follows:

1) A model-based parameter optimization trajectory tracking control scheme with velocity and torque constraints for wheeled mobile robots is proposed.

2) The model-based parameter optimization scheme proposed in this paper does not need to provide a given analytical time expression, and it only requires that the settling time is solvable.

3) This paper provides two optimization schemes. In the first scheme, the system torque maximum can be an accurate value when the sensor accuracy meets the requirements. And in the second scheme, the torque maximum can be in a saturation range when the sensor accuracy is limited.

4) In MATLAB simulation experiments, comparing with traditional backstepping control, fast terminal sliding mode control, and the model-based control [16], the parameter optimization control method presented in this paper is more effective to restrict the input torques and velocities with a reasonable settling time.

The rest of this paper is organized as follows. Section 2 introduces the mathematical model of the wheeled mobile robot. Section 3 discusses an improved modelbased controller with speed limitations. In Section 4, the model-based parameter optimization method with input torque and velocity limits is given. Section 5 studies the simulation experiments of three tracking trajectories by five control metohds. Section 6 is the discussion and comparison of the experimental results. Section 7 introduces the conclusions of this paper.

\section{Wheeled mobile robots model}

The designed differentially steered Wheeled Mobile Robot (WMR) is shown in Fig. $1, v_{v}=[v, \omega]^{T} \in \mathbb{R}^{2}, v$ and $\omega$ are the linear and angular velocities, respectively. $x \in \mathbb{R}$ and $y \in \mathbb{R}$ are the Cartesian coordinates of the mass center, and $\theta \in \mathbb{R}$ is the heading angle of the WMR shown in Fig. 1. There are some assumptions adopted in the WMR for the established trajectory tracking system model. To maintain the nonholonomic constraint, the WMR is not able to move sideways. The centroid of the WMR coincides with the geometric center [10]-[16].

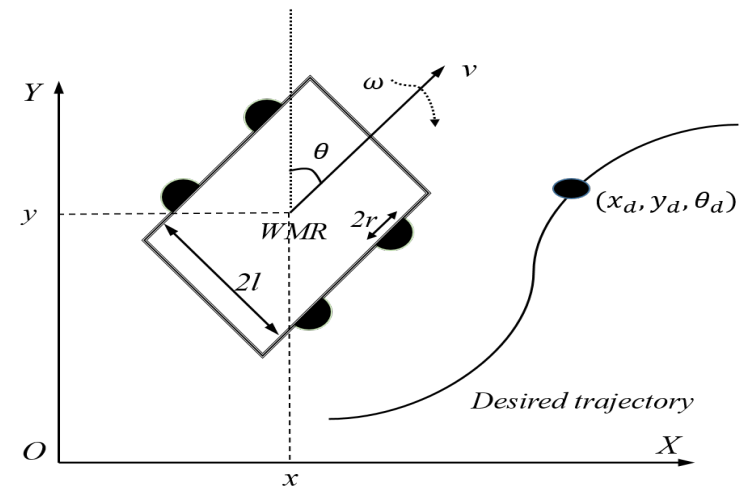

Fig. 1 Trajectory tracking of WMR with two independent driving wheels.

The Cartesian space kinematic equation (1) of the WMR configuration is as follows [1]-[2], [8]-[16]:

$\left\{\begin{array}{l}\dot{x}=-v \sin \theta \\ \dot{y}=v \cos \theta \\ \dot{\theta}=\omega\end{array}\right.$,

In Fig. 1, $r$ is the radius of the wheels, and the distance between the two rear wheels is $2 l . m$ is the mass of the WMR, the moment of inertia is $J . q=$ $[x, y, \theta]^{T} \in \mathbb{R}^{3}$ is the position vector $[1]-[2]$.

In this paper, the physical actuator velocity constraints are defined as [11]:

$\left\{\begin{array}{l}|v| \leq v_{c m 1}, v_{c m 1}>0 \\ |\omega| \leq \omega_{c m 1}, \omega_{c m 1}>0\end{array}\right.$.

The desired trajectory position vector is $q_{d}=\left[x_{d}, y_{d}\right.$, $\left.\theta_{d}\right]^{T}$ generated by

$\left\{\begin{array}{l}\dot{x_{d}}=-v_{d} \sin \theta_{d} \\ \dot{y_{d}}=v_{d} \cos \theta_{d} \\ \dot{\theta_{d}}=\omega_{d}\end{array}\right.$,

where $v_{d}$ and $\omega_{d}$ are desired velocity satisfying [11]

$\left\{\begin{array}{l}0<v_{d}<v_{c m 1} \\ 0<\omega_{d}<\omega_{c m 1}\end{array}\right.$

To meet the asymptotic stability of tracking the position vector $q$, the auxiliary velocity input is defined as $[32]$

$\left\{\begin{array}{l}v_{a i}=v_{d} \cos \theta_{e}+k_{1} x_{e} \\ \omega_{a i}=\omega_{d}+k_{2} v_{d} y_{e}+k_{3} v_{d} \sin \theta_{e}\end{array}\right.$, 
where $k_{1}, k_{2}$, and $k_{3}$ are adjustable positive constants, and $k_{2} \geq 1$.

The position tracking errors are [16]

$\left[\begin{array}{l}x_{e} \\ y_{e} \\ \theta_{e}\end{array}\right]=\left[\begin{array}{ccc}-\sin \theta & \cos \theta & 0 \\ -\cos \theta & -\sin \theta & 0 \\ 0 & 0 & 1\end{array}\right]\left[q_{d}-q\right]$.

And $[16]$

$\left\{\begin{array}{l}\dot{x}_{e}=y_{e} \omega-v+v_{d} \cos \theta_{e} \\ \dot{y}_{e}=-x_{e} \omega+v_{d} \sin \theta_{e} \\ \dot{\theta}_{e}=\omega_{d}-\omega\end{array}\right.$.

According to the Euler-Lagrange formulation, the dynamic equation of the WMR is described as [2]

$M(q) \ddot{q}=B(q) \tau+A^{T}(q) \lambda$,

where $\tau=\left[\tau_{1}, \tau_{2}\right]^{T} \in \mathbb{R}^{2 \times 1}$ is control input torque, $\lambda \in \mathbb{R}$ is a constraint vector, $M(q) \in \mathbb{R}^{3 \times 3}$ is a symmetric positive definite inertia matrix, $B(q) \in \mathbb{R}^{3 \times 2}$ is an input conversion matrix, and $A(q) \in \mathbb{R}^{1 \times 3}$ is a matrix related to nonholonomic constraints [2], [16]. $\tau_{1}$ and $\tau_{2}$ are the applied torques of two motors driving the two rear wheels, respectively. The torque constraints are defined as:

$\left\{\begin{array}{l}\left|\tau_{1}\right| \leq \tau_{1 m}, \tau_{1 m}>0 \\ \left|\tau_{2}\right| \leq \tau_{2 m}, \tau_{2 m}>0\end{array}\right.$

In the equation (8),

$M(q)=\left[\begin{array}{ccc}m & 0 & 0 \\ 0 & m & 0 \\ 0 & 0 & J\end{array}\right]$

$B(q)=\frac{1}{r}\left[\begin{array}{cc}-\sin \theta & -\sin \theta \\ \cos \theta & \cos \theta \\ l & -l\end{array}\right]$.

The nonholonomic constraint of the WMR can be written as $A^{T}(q) \dot{q}=0$. Define $H^{T}(q) A^{T}(q)=0$. Therefore, the following conclusions are established [16], [34]:

$\left\{\begin{array}{l}\dot{q}=H(q) v_{v} \\ \ddot{q}=H(q) \dot{v}_{v}+\dot{H}(q) v_{v}\end{array}\right.$,

where

$H(q)=\left[\begin{array}{cc}-\sin (\theta) & 0 \\ \cos \theta & 0 \\ 0 & 1\end{array}\right]$.

From the definitions of $H(q), M(q)$, it can be concluded that $H^{T}(q) M(q) \dot{H}(q)=0$. Under which, one has [16], [34]:

$$
\begin{aligned}
H^{T}(q) M(q)\left(H(q) \dot{v}_{v}+\dot{H}(q) v_{v}\right) & =H^{T}(q) B(q) \tau \\
+ & H^{T}(q) A^{T}(q) \lambda,
\end{aligned}
$$

$\bar{M}\left[\begin{array}{c}\dot{v} \\ \dot{\omega}\end{array}\right]=\bar{B}(q) \tau$

where $\bar{M}=\left[\begin{array}{cc}m & 0 \\ 0 & J\end{array}\right], \bar{B}=\frac{1}{r}\left[\begin{array}{cc}1 & 1 \\ l & -l\end{array}\right]$.

From the equations (8)-(15), the simplified dynamic control law model of the WMR is [33]-[34]

$\left\{\begin{array}{l}\dot{v}=\frac{\tau_{1}+\tau_{2}}{m r} \\ \dot{\omega}=\frac{\tau_{1}-\tau_{2}}{J r} l\end{array}\right.$.

\section{Improved model-based trajectory tracking controller}

In this part, a model-based improved control law scheme is addressed for the WMR to track the desired position trajectory with the velocity constraints.

From equations (4) and (9) in the literature [16], the improved control law is given as:

$\left[\begin{array}{c}\dot{v} \\ \dot{\omega}\end{array}\right]=-\left[\begin{array}{c}\rho_{1} \tilde{A}_{1} V_{11} \\ \rho_{2} \tilde{A}_{2} V_{12}\end{array}\right]+\left[\begin{array}{c}\left(v-v_{m p}\right) v_{a d} \\ \left(\omega-\omega_{m p}\right) \omega_{a d}\end{array}\right]$

where $\rho_{1}, \rho_{2}$ are adjustable positive constants.

Define [16]

$\tilde{A}_{i}=\left\{\begin{array}{r}\frac{1}{A_{i}}, A_{i} \neq 0 \\ 0, A_{i}=0\end{array}, i=1,2\right.$,

$\left[\begin{array}{l}A_{1} \\ A_{2}\end{array}\right]=\left[\begin{array}{c}v-v_{a i} \\ \omega-\omega_{a i}\end{array}\right]+\left[\begin{array}{c}\frac{\operatorname{sign}(v) h_{1}(v)}{v_{c m 1}-|v|} \\ \frac{\operatorname{sign}(\omega) h_{2}(\omega)}{\omega_{c m 1}-|\omega|}\end{array}\right]$.

Suppose [16]

$\left\{\begin{array}{l}h_{1}(v)=\left\{\begin{array}{l}1,|v|>v_{c m 2} \\ 0, \text { others }\end{array}\right. \\ h_{2}(\omega)=\left\{\begin{array}{l}1,|\omega|>\omega_{c m 2} \\ 0, \text { others }\end{array}\right.\end{array}\right.$,

where $\left[v_{c m 2}, \omega_{c m 2}\right]^{T}$ is a positive constant matrix, and $0<v_{c m 2}<v_{c m 1}, 0<\omega_{c m 2}<\omega_{c m 1}$.

$V_{1}$ is Lyapunov function, where [16]

$V_{1}=V_{11}+V_{12}$

$V_{11}=\frac{1}{2}\left(v-v_{a i}\right)^{2}+f_{1}(v)-\frac{1}{2}\left(v_{m p}-\right.$ $\left.v_{a i}\right)^{2}-f_{1}\left(v_{m p}\right)$

$V_{12}=\frac{1}{2}\left(\omega-\omega_{a i}\right)^{2}+f_{2}(\omega)-\frac{1}{2}\left(\omega_{m p}-\right.$ $\left.\omega_{a i}\right)^{2}-f_{2}\left(\omega_{m p}\right)$.

In $V_{1}, \frac{1}{2}\left(v-v_{a i}\right)^{2}$ is used for decreasing $\left\|v-v_{a i}\right\|$, and $\frac{1}{2}\left(\omega-\omega_{a i}\right)^{2}$ is employed to reduce $\left\|\omega-\omega_{a i}\right\|, f_{1}(v)$ is utilized to constraint linear velocity $v, f_{2}(\omega)$ is applied to limit angular velocity $\omega,-\frac{1}{2}\left(v_{m p}-v_{a i}\right)^{2}-f_{1}\left(v_{m p}\right)$ 
and $-\frac{1}{2}\left(\omega_{m p}-\omega_{a i}\right)^{2}-f_{2}\left(\omega_{m p}\right)$ are used to ensure that the minimum value of $V_{1}$ is 0 [16].

To achieve the aforementioned objective, let [16]

$$
\left\{\begin{array}{l}
f_{1}(v)=\left\{\begin{array}{l}
-\ln \frac{v_{c m 1}-|v|}{v_{c m 1}-v_{c m 2}},|v|>v_{c m 2} \\
0, \text { others }
\end{array}\right. \\
f_{2}(\omega)=\left\{\begin{array}{l}
-\ln \frac{\omega_{c m 1}-|\omega|}{\omega_{c m 1}-\omega_{c m 2}},|\omega|>\omega_{c m 2} \\
0, \text { others }
\end{array}\right.
\end{array}\right.
$$

$$
\left\{\begin{array}{l}
f_{1}\left(v_{m p}\right)=\left\{\begin{array}{l}
-\ln \frac{v_{c m 1}-\left|v_{m p}\right|}{v_{c m 1}-v_{c m 2}},\left|v_{m p}\right|>v_{c m 2} \\
0, \text { others }
\end{array}\right. \\
f_{2}\left(\omega_{m p}\right)=\left\{\begin{array}{l}
-\ln \frac{\omega_{c m 1}-\left|\omega_{m p}\right|}{\omega_{c m 1}-\omega_{c m 2}},\left|\omega_{m p}\right|>\omega_{c m 2} \\
0, \text { others }
\end{array} .\right.
\end{array}\right.
$$

Simultaneously, the following definitions should be established [16]:

$$
\begin{aligned}
& \left\{\begin{array}{l}
B_{1}=\frac{\left|v_{a i}\right|+v_{c m 1}-\sqrt{\left(\left|v_{a i}\right|-v_{c m 1}\right)^{2}+4}}{2} \\
B_{2}=\frac{\left|\omega_{a i}\right|+\omega_{c m 1}-\sqrt{\left(\left|\omega_{a i}\right|-\omega_{c m 1}\right)^{2}+4}}{2} \\
C_{1}=v_{c m 2}+\frac{1}{v_{c m 1}-v_{c m 2}} \\
C_{2}=\omega_{c m 2}+\frac{1}{\omega_{c m 1}-\omega_{c m 2}}
\end{array}\right. \\
& \left\{\begin{array}{l}
v_{m p}=\left\{\begin{array}{l}
\operatorname{sign}\left(v_{a i}\right) B_{1},\left|v_{a i}\right|>C_{1} \\
\operatorname{sign}\left(v_{a i}\right) v_{c m 2}, v_{c m 2}<\left|v_{a i}\right| \leq C_{1} \\
v_{a i},\left|v_{a i}\right| \leq v_{c m 2}
\end{array}\right. \\
\omega_{m p}=\left\{\begin{array}{l}
\operatorname{sign}\left(\omega_{a i}\right) B_{2},\left|\omega_{a i}\right|>C_{2} \\
\operatorname{sign}\left(\omega_{a i}\right) \omega_{c m 2}, \omega_{c m 2}<\left|\omega_{a i}\right| \leq C_{2} \\
\omega_{a i},\left|\omega_{a i}\right| \leq \omega_{c m 2}
\end{array}\right.
\end{array}\right.
\end{aligned}
$$

Assume

$\operatorname{sign}(x)=\left\{\begin{array}{l}1, x>0 \\ 0, x=0 \\ -1, x<0\end{array}\right.$.

In this paper, defining $v_{a d}$ and $\omega_{a d}$ in equation (17) are as follows:

$$
\left\{\begin{array}{l}
v_{a d}=\dot{v}_{a i}+v_{d} \omega_{d} \sin \theta_{e}-k_{1} v_{d} \cos \theta_{e} \\
\omega_{a d}=\dot{\omega}_{a i}-v_{d}\left(k_{2} v_{d} \sin \theta_{e}+k_{3} \omega_{d} \cos \theta_{e}\right)
\end{array} .\right.
$$

For $|v|<v_{c m 1},|\omega|<\omega_{c m 1}$, if $v \rightarrow v_{c m 1}$ and $\omega \rightarrow$ $\omega_{c m 1}$, it has $V_{1} \rightarrow \infty$. Lyapunov function (21) helps the designed controller to constrain velocities [16].

The detailed proof process in the literature [16] demonstrated that using the control law (17) with the velocity constraints $|v|<v_{c m 1},|\omega|<\omega_{c m 1},\left|v_{a i}\right|<v_{c m 2},\left|\omega_{a i}\right|$ $<\omega_{c m 2}$, the velocity and position errors of the WMR system would converge to zero. The closed-loop control system is asymptotically stable, and the system states would not violate the constraints [16].

Supplement: The mian difference between this paper and the literature [16] is formula (29), helping the controller to achieve stably error-free tracking. The first control law in [16] referred to in this paper is the modelbased control for the system with known model and parameters. The second control law on the title of [16] not mentioned in this paper is for the system having an unknown model or parameter.

\section{Model-based parameter optimization control}

The improved model-based control method realize the error-free tracking of the position and velocity vectors quickly with the speed constraints. This paper wants to achieve the non-error track combined with the speed and torque constraints. Adding torque constraints to the system may prolong the settling time. Therefore, we addressed a model-based parameter optimization control scheme to decrease the increasing settling time by the torque constraints.

The gradient descent method updates iteratively along the negative gradient direction of the objective function and finally converges to the minimum point. Cauchy proposed the gradient descent method in 1847. Due to the simple concept, the gradient descent method has become one of the most commonly used parameter minimization methods [29]-[31]. The basic idea of this method is to assume that the gradient vector is in the steepest ascending direction and take a sufficiently small step in the opposite direction, which causes the function value to be lower than the previous value. Repeat this process to reach the minimum value of the function.

The continuous gradient descent function is

$\dot{x}=-\varepsilon \nabla f(x)$,

where, $\varepsilon>0$ is the step size. $\nabla f(x)$ represents the gradient of $f(x)$ at $x$.

The corresponding discrete gradient descent function is

$x(a+1)=x(a)-\varepsilon \nabla f(x(a))$,

where $a$ is the number of iteration steps, and $x(a)$ is the $a$-th step variable value. The gradient descent method is an iterative optimization algorithm for obtaining an approximate solution from the next step of the current step until reaching the termination condition in a stepwise loop.

From equation (16), we can conclude

$$
\left[\begin{array}{l}
\tau_{1} \\
\tau_{2}
\end{array}\right]=\left[\begin{array}{l}
\frac{1}{2} r\left(m \dot{v}+\frac{J}{l} \dot{\omega}\right) \\
\frac{1}{2} r\left(m \dot{v}-\frac{J}{l} \dot{\omega}\right)
\end{array}\right] .
$$

In the equations (1)-(29), the all adjustable parameters are $k_{1}, k_{2}, k_{3}, \rho_{1}, \rho_{2}$. And they are all constants greater than zero. Define $\zeta=\left[k_{1}, k_{2}, k_{3}, \rho_{1}, \rho_{2}\right]^{T} \in\left(\mathbb{R}^{+}\right)^{5}$, 


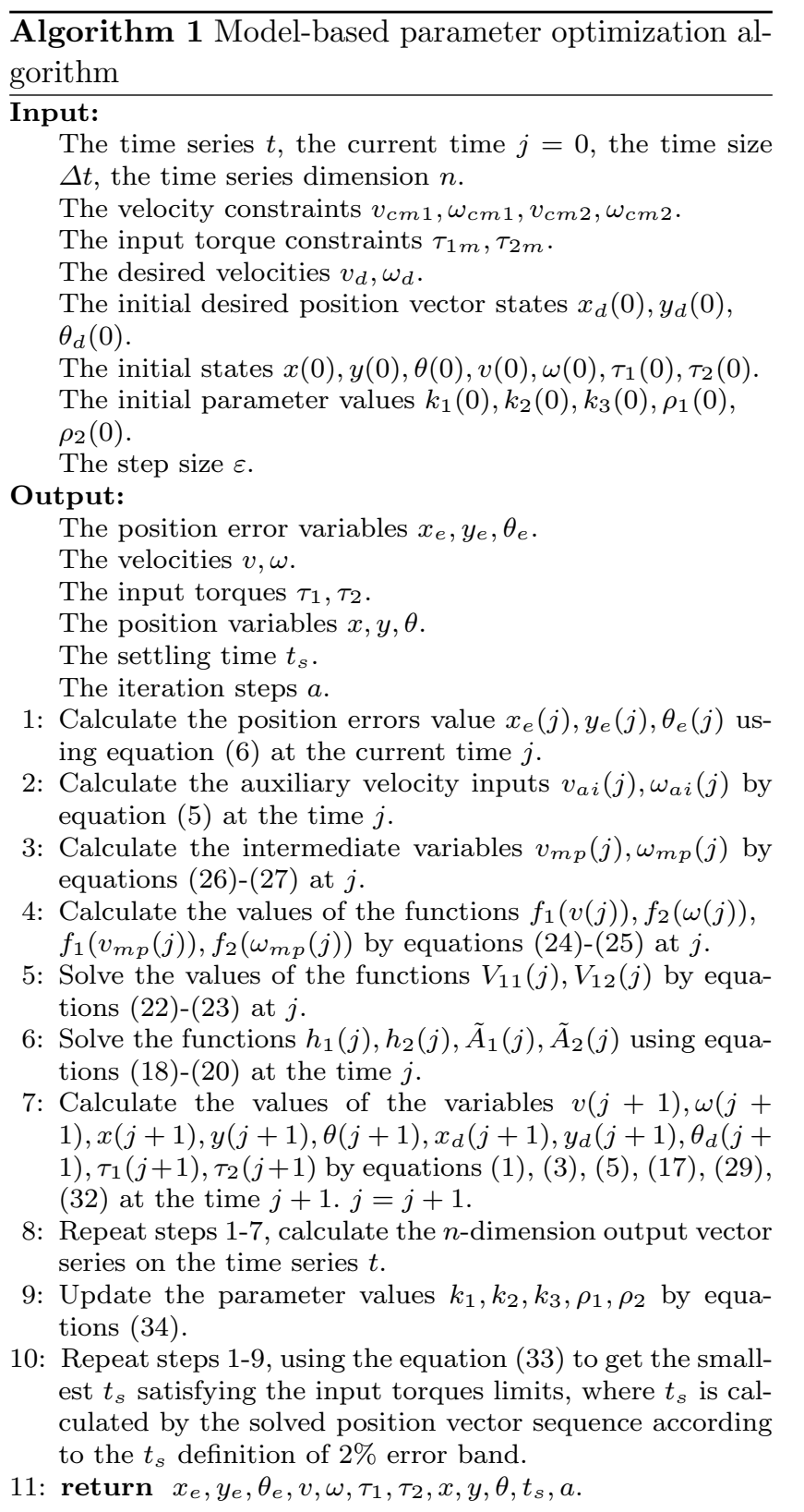

where $k_{2} \geq 1$. The optimization goal is to minimize settling time $t_{s}$, when the input torques are limited by $\left|\tau_{1}\right| \leq \tau_{1 m},\left|\tau_{2}\right| \leq \tau_{2 m} \cdot t_{s 1}, t_{s 2}, t_{s 3}$ are the settling times of the position errors $x_{e}, y_{e}, \theta_{e}$, respectively. Define $t_{s}=\max \left(t_{s 1}, t_{s 2}, t_{s 3}\right)$.

For keeping $\left|\tau_{1}\right| \leq \tau_{1 m},\left|\tau_{2}\right| \leq \tau_{2 m}, t_{s}$ may increase to cause the position error series $x_{e}, y_{e}, \theta_{e}$ to fail to converge to zero within the limited working time $t$. In practical applications, we usually hope that the settling time $t_{s}$ is minimal.

Set the optimal objective function as

$$
\xi^{*}=\underset{\left|\tau_{1}\right| \leq \tau_{1 m},\left|\tau_{2}\right| \leq \tau_{2 m}, \xi \in \zeta}{\arg \min }\left(t_{s}\right)
$$

The adjustable parameter optimization formula obtained by the gradient descent method is

$$
\left\{\begin{array}{l}
k_{1}(a+1)=k_{1}(a)-\varepsilon \nabla f\left(k_{1}(a)\right) \\
k_{2}(a+1)=k_{2}(a)-\varepsilon \nabla f\left(k_{2}(a)\right) \\
k_{3}(a+1)=k_{3}(a)-\varepsilon \nabla f\left(k_{3}(a)\right) \\
\rho_{1}(a+1)=\rho_{1}(a)-\varepsilon \nabla f\left(\rho_{1}(a)\right) \\
\rho_{2}(a+1)=\rho_{2}(a)-\varepsilon \nabla f\left(\rho_{2}(a)\right)
\end{array} .\right.
$$

Using Algorithm 1 Model-based parameter optimization algorithm, we can get the smallest possible settling time $t_{s}$ satisfying the limits $\left|\tau_{1}\right| \leq \tau_{1 m a} \leq \tau_{1 m},\left|\tau_{2}\right| \leq$ $\tau_{2 m a} \leq \tau_{2 m}$. In this paper, $\tau_{1 m a}=\max \left(\left|\tau_{1}\right|\right), \tau_{2 m a}=$ $\max \left(\left|\tau_{2}\right|\right)$.

Since this strategy uses the model-based controller with speed limitation, the model-based parameter optimization method has both the speed and torque constraints, and its settling time can be the smallest. Furthermore, in Algorithm 1, the optimization strategy only needs the settling time to be solvable and does not need to have an analytic expression.

\section{Simulations and experiments}

In this section, we did the MATLAB simulation experiments to compare the backstepping control, the fast terminal sliding mode control, the improved model-based control, and the parameter optimization control scheme proposed in the paper to track different trajectories. The backstepping control, the fast terminal sliding mode control, and the model-based control methods are effective control methods for robots to achieve trajectory tracking. This section utilizes these three methods to carry out simulation comparison experiments to verify the effectiveness of the method proposed in this paper.

In this section, the control parameters are chosen as $c_{1}=c_{2}=5$ for the backstepping control. For the fast terminal sliding mode control, the parameters are $\alpha_{1}=\alpha_{2}=2, \beta_{1}=\beta_{2}=1, p_{1}=p_{2}=83, q_{1}=q_{2}=85$. For the improved model-based control, the parameters are chosen as $k_{1}=2, k_{2}=8, k_{3}=10, \rho_{1}=\rho_{2}=1$ and $v_{c m 1}=1.5 \mathrm{~m} / \mathrm{s}, v_{c m 2}=1.3 \mathrm{~m} / \mathrm{s}, \omega_{c m 1}=1 \mathrm{rad} / \mathrm{s}$ $\omega_{c m 2}=0.8 \mathrm{rad} / \mathrm{s}$.

Considering a four-wheeled mobile robot shown in Fig. $1, m=50 \mathrm{~kg}, J=5.4 \mathrm{kgm}^{2}, l=250 \mathrm{~mm}, r=100 \mathrm{~mm}$, $g=9.8 \mathrm{~m} / \mathrm{s}^{2} . t \in\left[0, t_{m}\right], t_{m}=80 \mathrm{~s}$, time size $t_{l}=0.01 \mathrm{~s}$. The initial values are $q_{(0)}=[-0.5,-0.5,-0.7]^{T}, q_{d(0)}=$ $[0,0,-\pi / 2]^{T}, v_{(0)}=0 \mathrm{~m} / \mathrm{s}, \omega_{(0)}=0 \mathrm{rad} / \mathrm{s}, \tau_{1(0)}=0 \mathrm{Nm}$, $\tau_{2(0)}=0 \mathrm{Nm}$. To track the trajectories, the input torques are according to equation (32).

In Table 1-Table3, $x_{e m}=\max \left(\left|x_{e}\right|\right), y_{e m}=\max \left(\left|y_{e}\right|\right)$ $, \theta_{e m}=\max \left(\left|\theta_{e}\right|\right), v_{m}=\max (|v|), \omega_{m}=\max (|\omega|)$. 
Table 1 Circle trajectory tracking comparison

\begin{tabular}{lllllllll}
\hline Control scheme & $x_{e m}[\mathrm{~m}]$ & $y_{e m}[\mathrm{~m}]$ & $\theta_{e m}[\mathrm{rad}]$ & $v_{m}[\mathrm{~m} / \mathrm{s}]$ & $\omega_{m}[\mathrm{rad} / \mathrm{s}]$ & $\tau_{1 m a}[\mathrm{Nm}]$ & $\tau_{2 m a}[\mathrm{Nm}]$ & $t_{s}[\mathrm{~s}]$ \\
\hline Backstepping & 0.706 & 0.062 & 0.871 & 3.504 & 0.100 & 678.668 & 1069.739 & 17.050 \\
Sliding mode & 0.706 & 0.101 & 0.871 & 3.274 & 2.262 & 572.725 & 1061.061 & 38.090 \\
Model-based & 0.718 & 0.203 & 0.871 & 0.834 & 0.696 & 2.035 & 2.466 & 17.080 \\
Optimal scheme1 & 0.740 & 0.096 & 0.871 & 0.896 & 0.529 & 1.870 & 2.000 & 11.090 \\
Optimal scheme2 & 0.744 & 0.116 & 0.871 & 0.900 & 0.531 & 1.894 & 1.986 & 13.040 \\
\hline
\end{tabular}

5.1 Circle trajectory tracking
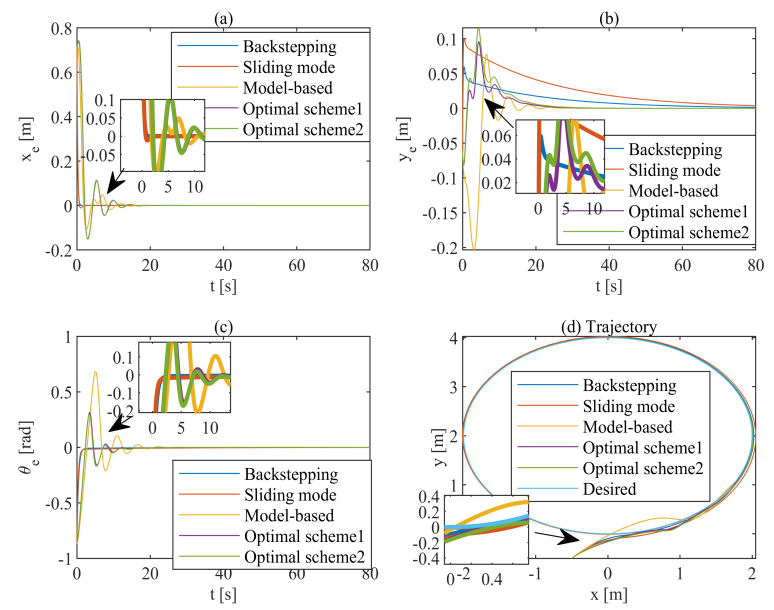

Fig. 2 Simulation result 1 of the circle trajectory tracking.
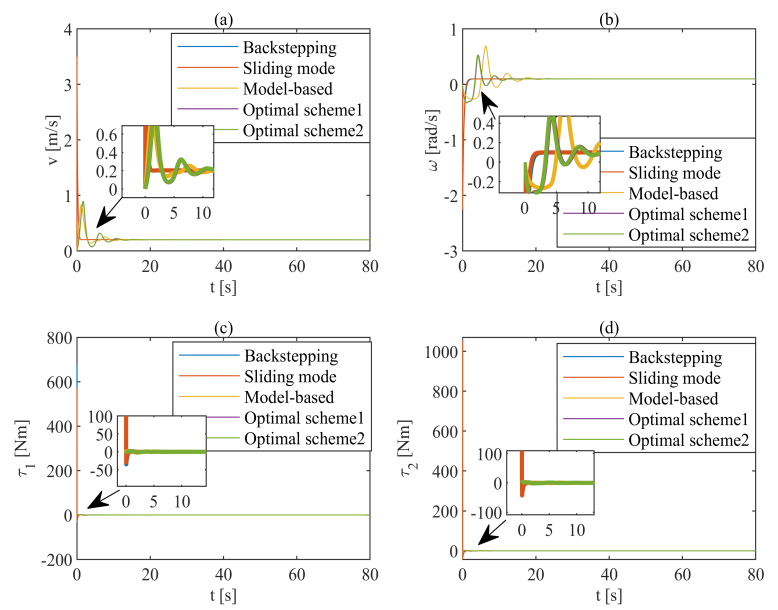

Fig. 3 Simulation result 2 of the circle trajectory tracking.

In this part, we studied circular trajectory tracking experiments. The experiment data of Fig. 2-Fig. 3 are shown in Table 1. The desired velocities are given as $v_{d}=0.2 \mathrm{~m} / \mathrm{s}, \omega_{d}=0.1 \mathrm{rad} / \mathrm{s}$.
Using Algorithm 1, the adjustable parameters of the model-based parameter optimal scheme 1 are $k_{1}=$ $2.662004181965706, k_{2}=8.846506030156295, k_{3}=11.4$ $97581737627470, \rho_{1}=0.329043268812957, \rho_{2}=1.55163$ 6615967679 . And for the optimal scheme 2 , the parameters are $k_{1}=2.7, k_{2}=8.8, k_{3}=11.5, \rho_{1}=0.3, \rho_{2}=1.6$ based on the optimal scheme 1 .

Fig. 2(a)-(c) shows the position errors. Fig. 2(d) displays the tracking and desired circle trajectories. Fig. $3(\mathrm{a})$ and (b) show the linear and angular velocities. The input torques are given in Fig. 3(c) and (d).
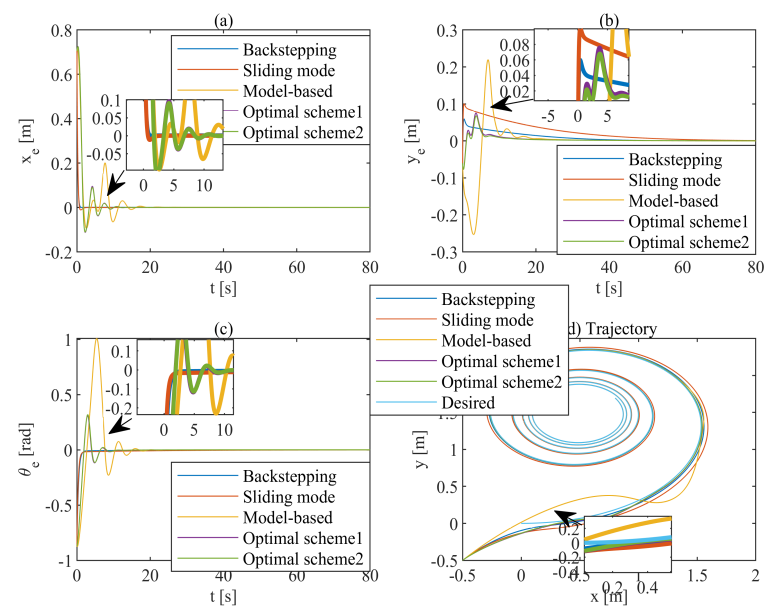

Fig. 4 Simulation result 1 of the spiral line trajectory tracking.

\subsection{Spiral line trajectory tracking}

In this part, spiral line trajectory tracking simulation experiments are carried out. The experiment data of Fig. 4-Fig. 5 are shown in Table 2. The desired velocities are given as $v_{d}=0.2+0.001 t, \omega_{d}=0.1+0.008 t$.

For the optimal scheme 1, the parameters are solved as $k_{1}=3.686967461306886, k_{2}=7.96746438723441$ $0, k_{3}=9.931440380829557, \rho_{1}=0.491122644557975, \rho_{2}$ $=2.044105956253549$. The parameters are $k_{1}=3.7, k_{2}$ $=8.0, k_{3}=9.9, \rho_{1}=0.5, \rho_{2}=2.0$ for the optimal scheme 2 . 
Table 2 Spiral line trajectory tracking comparison

\begin{tabular}{lllllllll}
\hline Control scheme & $x_{e m}[\mathrm{~m}]$ & $y_{e m}[\mathrm{~m}]$ & $\theta_{e m}[\mathrm{rad}]$ & $v_{m}[\mathrm{~m} / \mathrm{s}]$ & $\omega_{m}[\mathrm{rad} / \mathrm{s}]$ & $\tau_{1 \mathrm{ma}}[\mathrm{Nm}]$ & $\tau_{2 m a}[\mathrm{Nm}]$ & $t_{s}[\mathrm{~s}]$ \\
\hline Backstepping & 0.706 & 0.062 & 0.871 & 3.504 & 0.740 & 678.668 & 1069.739 & 14.680 \\
Sliding mode & 0.706 & 0.101 & 0.871 & 3.274 & 2.262 & 572.725 & 1061.061 & 32.700 \\
Model-based & 0.714 & 0.253 & 1.009 & 0.827 & 0.787 & 1.844 & 3.113 & 14.600 \\
Optimal scheme1 & 0.726 & 0.077 & 0.871 & 0.961 & 0.740 & 2.463 & 3.000 & 6.330 \\
Optimal scheme2 & 0.725 & 0.077 & 0.871 & 0.962 & 0.740 & 2.476 & 3.020 & 7.350 \\
\hline
\end{tabular}
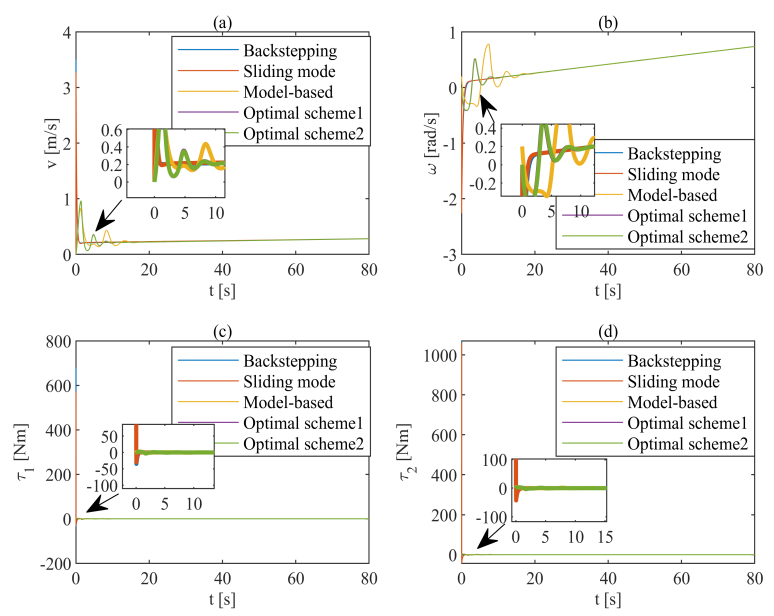

Fig. 5 Simulation result 2 of the spiral line trajectory tracking.

Fig. 4(a)-(c) shows the position errors of tracking spiral line trajectory. Fig. 4(d) displays the spiral line trajectories. Fig. 5(a) and (b) are the linear and angular velocities. Fig. 5(c) and (d) are the input torques.
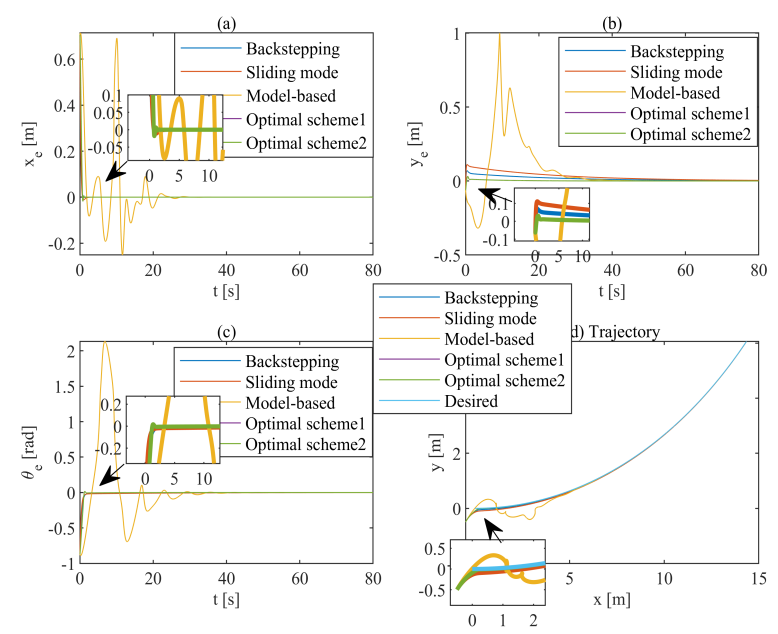

Fig. 6 Simulation result 1 of the parabolic line trajectory tracking.
5.3 Parabolic line trajectory tracking
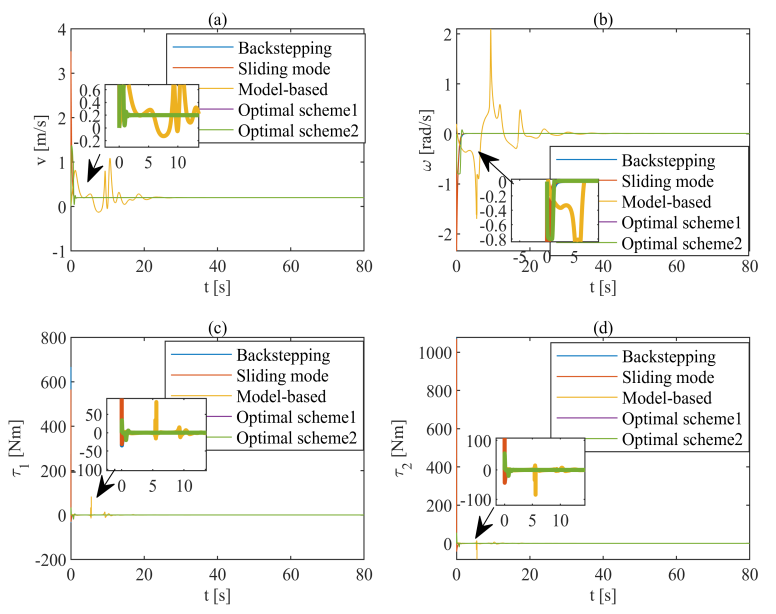

Fig. 7 Simulation result 2 of the parabolic line trajectory tracking.

In this part, parabolic line trajectory tracking experiments are performed. The experiment data is shown in Fig. 6, Fig. 7, and Table 3. The desired velocities are $v_{d}=0.2 \mathrm{~m} / \mathrm{s}, \omega_{d}=0.01 \mathrm{rad} / \mathrm{s}$.

For the optimal scheme 1 , the parameters are $k_{1}=$ $16.664718729426582, k_{2}=12.347534665360824, k_{3}=25$ $.054244919610280, \rho_{1}=13.378510292167398, \rho_{2}=20.4$ 15445695047660. The parameters are chosen as $k_{1}=$ $16.7, k_{2}=12.3, k_{3}=25.1, \rho_{1}=13.4, \rho_{2}=20.4$ for the optimal scheme 2 .

Fig. 6(a)-(c) are the position errors. Fig. 6(d) shows the tracking trajectories and the desired trajectory. Fig. 7(a) and (b) show the tracking linear velocity and angular velocity. The tracking input torques are shown in Fig. 7(c) and (d).

\section{Discussion}

From Fig. 2-Fig. 7 and Table 1-Table 3, it can be observed that all the five control schemes can track the three desired trajectories, and the error signals can converge to a small neighborhood of zero. However, from 
Table 3 Parabolic line trajectory tracking comparison

\begin{tabular}{|c|c|c|c|c|c|c|c|c|}
\hline Control scheme & $x_{e m}[m]$ & $y_{e m}[m]$ & $\theta_{e m}[r a d]$ & $v_{m}[\mathrm{~m} / \mathrm{s}]$ & $\omega_{m}[\mathrm{rad} / \mathrm{s}]$ & $\tau_{1 m a}[N m]$ & $\tau_{2 m a}[N m]$ & $t_{s}[s]$ \\
\hline Backstepping & 0.706 & 0.070 & 0.871 & 3.496 & 0.010 & 667.025 & 1077.537 & 24.050 \\
\hline Sliding mode & 0.706 & 0.111 & 0.871 & 3.280 & 2.340 & 565.395 & 1070.770 & 40.660 \\
\hline Model-based & 0.885 & 1.060 & 2.186 & 1.102 & 2.115 & 118.796 & 119.307 & 33.330 \\
\hline Optimal scheme1 & 0.706 & 0.062 & 0.871 & 1.377 & 0.085 & 33.612 & 56.000 & 1.140 \\
\hline Optimal scheme 2 & 0.706 & 0.062 & 0.871 & 1.377 & 0.086 & 33.693 & 56.075 & 1.140 \\
\hline
\end{tabular}

Fig. 2-Fig. 7 and Table 1-Table 3, we can observe the following.

(A). The latter three control methods can better limit the absolute maximum value of the speeds and torques compared with the first two traditional control methods. The maximum torques acquired by the first two classic control algorithms are too large, which leads to the large size and high cost of the motor.

(B). The latter two model-based parameter optimization schemes, compared with the improved modelbased control method, realize the optimization of the settling time and the overall rapidity. Moreover, the model-based parameter optimization schemes reduce the oscillation amplitude that appears in the improved modelbased control method and improves the riding index of the control system.

(C). The model-based parameter optimal scheme 1 can strictly limit the maximum absolute value of the torque to a small value (e.g. $2.000 \mathrm{Nm}$ in Table $1,3.000 \mathrm{Nm}$ in Table 2, and $56.000 \mathrm{Nm}$ in Table 3) through the precise setting of the parameters. When the accuracy of the robot sensor meets the parameter setting requirements, this optimization scheme can achieve strict control of the absolute maximum value of the torque.

(D). The optimal scheme 2 also realizes the limitation of the speed, torque, and settling time, and its requirements on the sensor accuracy are much lower than that of optimal scheme 1. The adjustable parameters in optimal scheme 1 need to keep 15 digits after the decimal point, while the optimal scheme 2 only needs to keep 1 digit after the decimal point. Compared with optimal scheme 1 , optimal scheme 2 can be widely applicable to more environments and has lower application costs.

The experiment data of the circle, spiral line, and parabolic line trajectories in Table 1-3 are averaged to obtain comparison histograms shown in Fig. 8-Fig. 10. The data in Table 1-3 are the absolute maximum values of the indexes. Averaging the data in Table 1-3 is solving the absolute maximum mean value of the experimental indexes. Fig. 8 shows the comparison of the average values of the position errors of three trajectories. The comparison of the absolute maximum mean value of velocities and settling time of three trajecto-

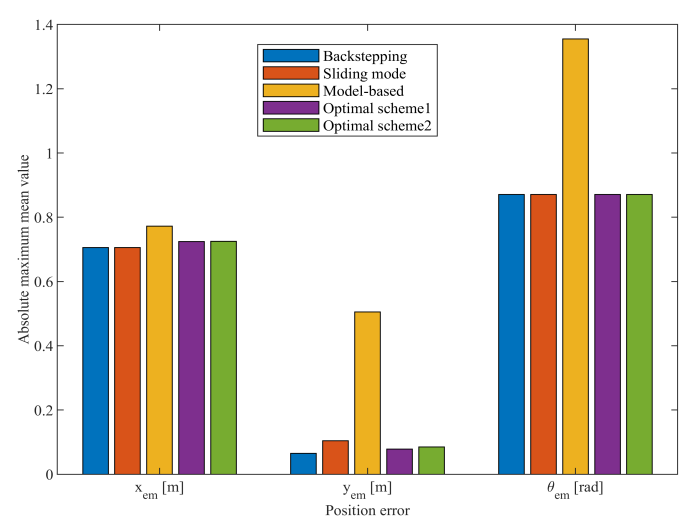

Fig. 8 Comparison of the absolute maximum mean value of position error of three trajectories.

ries are given in Fig. 9. Fig. 10 displays the comparison of the absolute maximum mean value of torques of three trajectories. The following conclusions can be obtained from the analysis of the three comparison histograms.

(1). The position error absolute maximum mean values of the improved model-based control algorithm are the largest. The absolute maximum mean values of the position error of the other four algorithms only have little difference.

(2). The absolute maximum mean value of the linear velocity of the backstepping algorithm is the largest, and the absolute maximum mean value of the angular velocity of the sliding mode algorithm is the largest. The maximum absolute mean values of linear and angular velocities obtained by the model-based parameter optimization algorithms are relatively small.

(3). The maximum absolute mean value of the settling time of sliding mode control is the largest, and the maximum absolute mean values of the settling time of the optimization algorithms proposed in this paper are the smallest.

(4). The maximum absolute mean values of the torques of the backstepping method are the largest, and the maximum absolute mean values of the torques of the optimization algorithms proposed in this paper are the smallest.

Judging from the experimental curves and data of the torques, settling time, speed, and position error of 


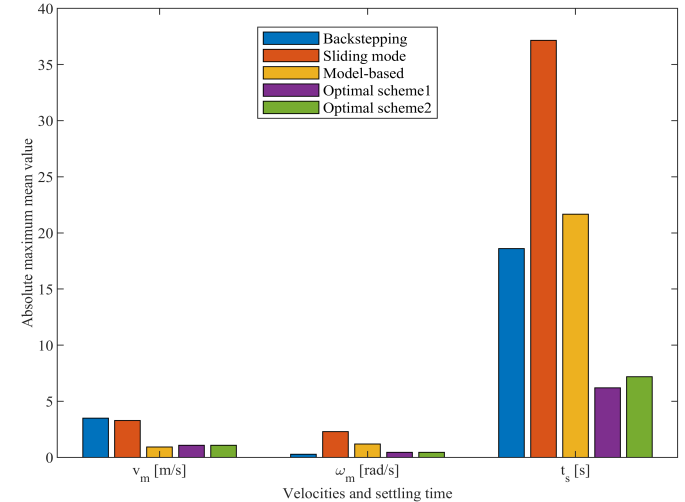

Fig. 9 Comparison of the absolute maximum mean value of velocities and settling time of three trajectories.

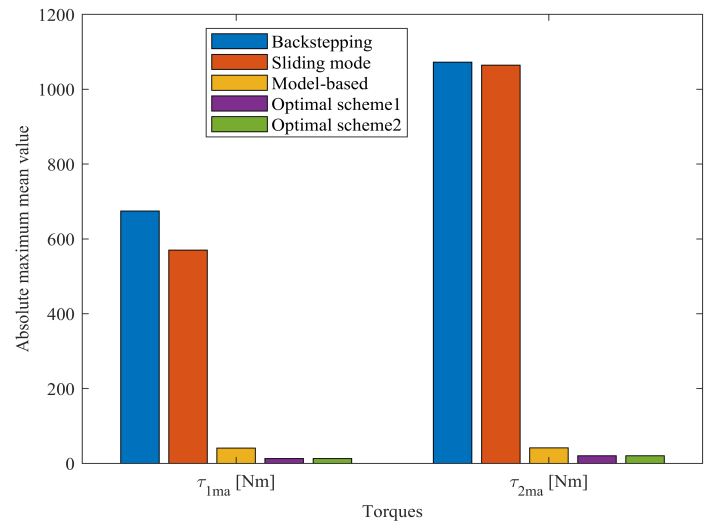

Fig. 10 Comparison of the absolute maximum mean value of torques of three trajectories.

the five algorithms, the model-based parameter optimization algorithm proposed in this paper is the best. And the other three algorithms have their advantages and disadvantages under different indicators.

\section{Conclusion}

Regarding the above research of trajectory tracking, the control torque and velocity obtained based on the first two traditional classic trajectory tracking control algorithms may cause large initial values. We design a model-based parameter optimization algorithm with torque and velocity constraints. Constraining the torque and velocity makes this proposed method succeeds in settling down the problems of oversaturation, mechanical size constraints, and energy utilization efficiency caused by excessive torque and speed of the motor.

The limitation of the torque and velocity will cause the rapidity index to deteriorate. The model-based parameter optimization method introduced in this paper performs torque and velocity constraints while minimizing the weakening of the rapidity. This research method has achieved a satisfying balance between the size of the input torque and velocity values and the size of the settling time value, which has a great practical application value.

The majority of the time optimization methods need to provide an analytical expression of the system time, while this parameter time optimization strategy only needs that the system time can be solvable. Only needing the system time can be solvable makes this time optimization scheme easier to implement and solve.

Furthermore, the first three methods can only use the trial and error method or the empirical method to set the adjustable control parameters. The model-based parameter optimization method proposed in this paper presents design schemes and solutions to select adjustable control parameters through iterative learning based on parameter initial setting values.

In the research of this paper, we caught sight of that the model-based parameter optimization method sometimes traps in the local optima. The next step of our work plan is to seek a control method to solve the globally optimal value.

\section{References}

1. Yang, J., Kim, J.: Sliding mode control for trajectory tracking of nonholonomic wheeled mobile robots. In: IEEE Trans. on Robotics and Automation, 15(3), pp. 578-587 (1999), https://doi.org/10.1109/70.768190

2. Lan, Y., Chen, X.: Trajectory tracking system of wheeled robot based on immune algorithm and sliding mode variable structure. Intel Serv Robotics. (2020). https://doi.org/10.1007/s11370-020-00325-8

3. Zhang, Hj., Gong, Jw., Jiang, Y. et al: An iterative linear quadratic regulator based trajectory tracking controller for wheeled mobile robot. J. Zhejiang Univ. -Sci., 13, 593-600 (2012). https://doi.org/10.1631/jzus.C1100379

4. Taghavifar, H., Rakheja, S.: A novel terramechanics-based path-tracking control of terrain-based wheeled robot vehicle with matched-mismatched uncertainties. IEEE Transactions on Vehicular Technology. 69(1), 67-77 (2020). https://doi.org/10.1109/TVT.2019.2950288

5. Dian, S. et al.: Modeling and trajectory tracking control for magnetic wheeled mobile robots based on improved dual-heuristic dynamic programming. IEEE Transactions on Industrial Informatics. 17(2) , 1470-1482 (2021). https://doi.org/10.1109/TII.2020.2983841

6. Khan, H., Iqbal, J., Baizid, K. et al: Longitudinal and lateral slip control of autonomous wheeled mobile robot for trajectory tracking. Frontiers Inf Technol Electronic Eng., 16, 166-172 (2015). https://doi.org/10.1631/FITEE.1400183

7. Binh, N.T., Tung, N.A., Nam, D.P. et al.: An adaptive backstepping trajectory tracking control of a tractor trailer wheeled mobile robot. Int. J. Control Autom. Syst., 17, 465-473 (2019). https://doi.org/10.1007/s12555-017-07110 
8. Li, IH., Chien, YH., Wang, WY. et al: Hybrid intelligent algorithm for indoor path planning and trajectory-tracking control of wheeled mobile robot. Int. J. Fuzzy Syst., 18, 595-608 (2016). https://doi.org/10.1007/s40815-016-01660

9. Li, Z., Deng, J., Lu, R., Xu, Y., Bai, J., Su, C.: Trajectory-tracking control of mobile robot systems incorporating neural-dynamic optimized model predictive approach. IEEE Transactions on Systems, Man, and Cybernetics: Systems. 46(6), 740-749 (2016). https://doi.org/10.1109/TSMC.2015.2465352

10. Abdelwahab, M., Parque, V., Fath Elbab, A.M.R., Abouelsoud, A.A., Sugano, S.: Trajectory tracking of wheeled mobile robots using z-number based fuzzy logic. IEEE Access. 8, 18426-18441 (2020). https://doi.org/10.1109/ACCESS.2020.2968421

11. Yu, X., Liu, L., Feng, G.: Trajectory Tracking for Nonholonomic Vehicles with Velocity Constraints. IFAC-PapersOnLine. 48(11 11) , pp. 918-923 (2015). https://doi.org/10.1016/j.ifacol.2015.09.3084

12. Liu, S. et al.: Trajectory tracking for leaderfollower vehicle system with velocity constraints. In: IEEE International Conference on Robotics and Biomimetics (ROBIO), vol.283, pp.474-479 (2018) https://doi.org/10.1109/ROBIO.2018.8665096

13. Yang, H., Guo, M., Xia, Y., Cheng, L.: Trajectory tracking for wheeled mobile robots via model predictive control with softening constraints. IET Control Theory Appl., 12, 206-214 (2018). https://doi.org/10.1049/iet-cta.2017.0395

14. Guo, C., Sun, Z., Chen, Y., Xie, Y., Li, S., Qian, H.: Trajectory tracking of unicycle-type robots with constraints. In: IEEE International Conference on Robotics and Biomimetics (ROBIO), pp. 1700-1705 (2018). https://doi.org/10.1109/ROBIO.2018.8665273

15. Xu, Z., Yang, S.X., Gadsden, S.A.: Enhanced bioinspired backstepping control for a mobile robot with unscented kalman filter. IEEE Access. 8, 125899-125908 (2020). https://doi.org/10.1109/ACCESS.2020.3007881

16. Chen, Z., Liu, Y., He, W., Qiao, H., Ji, H.: Adaptive neural network-based trajectory tracking control for a nonholonomic wheeled mobile robot with velocity constraints. IEEE Trans. Ind. Electron. (2020) https://doi.org/10.1109/tie.2020.2989711

17. Ailon, A., Arogeti, S.: Control of WMRs with dynamic models subject to bounded inputs *. In: 29th Mediterranean Conference on Control and Automation (MED), pp. 735-740 (2021). https://doi.org/10.1109/MED51440.2021.9480308

18. Wang, D., Wei, W., Wang, X. et al.: Formation control of multiple mecanum-wheeled mobile robots with physical constraints and uncertainties. Appl Intell. (2021). https://doi.org/10.1007/s10489-021-02459-3

19. Yue, M., An, C. Sun, JZ.: An efficient model predictive control for trajectory tracking of wheeled inverted pendulum vehicles with various physical constraints. Int. J. Control Autom. Syst. 16, 265-274 (2018). https://doi.org/10.1007/s12555-016-0393-z

20. Verscheure, D., Demeulenaere, B., Swevers, J., De Schutter, J., Diehl, M.: Time-energy optimal path tracking for robots: a numerically efficient optimization approach. In: 10th IEEE International Workshop on Advanced Motion Control, pp. 727-732 (2008). https://doi.org/10.1109/AMC.2008.4516157

21. Van Willigenburg, L.G.: Computation and implementation of digital time-optimal feedback controllers for an industrial $\mathrm{X}-\mathrm{Y}$ robot subjected to path, torque, and velocity constraints. International
Journal of Robotics Research. 12 (5), 420-433 (1993). https://doi.org/10.1109/iccwamtip47768.2019.9067568

22. Li, L., Xiao, J., Zou, Y., Zhang, T.: Time-optimal path tracking for robots a numerical integration-like approach combined with an iterative learning algorithm. Industrial Robot. 46(6), 763-778 (2019). https://doi.org/10.1108/IR03-2019-0063

23. Verscheure, D., Diehl, M., De Schutter, J., Swevers, J.: Recursive log-barrier method for online time-optimal robot path tracking. In: American Control Conference, pp. pp. 4134-4140 (2009). https://doi.org/10.1109/ACC.2009.5159938

24. Dong, W., Ding, Y., Huang, J., Zhu, X., Ding, H.: An efficient approach of time-optimal trajectory generation for the fully autonomous navigation of the quadrotor. ASME. J. Dyn. Sys., Meas., 139(6), 060102 (2017). https://doi.org/10.1115/1.4035453

25. Steinhauser, A., Swevers, J.: Iterative learning of feasible time-optimal trajectories for robot manipulators. industrial robot. IFAC PapersOnline. 50(1), 12095-12100 (2017). https://doi.org/10.1016/j.ifacol.2017.08.2123

26. Palleschi, A., Garabini, M., Caporale, D., Pallottino, L.: Time-optimal path tracking for jerk controlled robots. IEEE Robotics and Automation Letters. 4(4), 3932-3939 (2019). https://doi.org/10.1109/LRA.2019.2929979

27. Zhang, Q., Li, S., Guo, J., Gao, X.: Time-optimal path tracking for robots under dynamics constraints based on convex optimization. Robotica. 34(9), 2116-2139 (2016). https://doi.org/10.1017/S0263574715000247

28. Zhang, T., Zhang, M., Zou, Y.: Time-optimal and smooth trajectory planning for robot manipulators. Int. J. Control Autom. Syst., 19, 521-531 (2021). https://doi.org/10.1007/s12555-019-0703-3

29. Zubair, S., Singha, A.K.: Parameter optimization in convolutional neural networks using gradient descent. In: Chaudhary, A., Choudhary, C., Gupta, M., Lal, C., Badal, T. (eds) Microservices in Big Data Analytics. Springer, Singapore. (2020). https://doi.org/10.1007/978-981-15-0128$9 \_8$

30. Huynh, B.-P., Wu, C.-W., Kuo, Y.-L.: Force/position hybrid control for a hexa robot using gradient descent iterative learning control algorithm. IEEE Access. 7, 72329-72342 (2019). https://doi.org/10.1109/ACCESS.2019.2920020

31. Shikalgar, A., Sonavane,S.: An enhanced stochastic gradient descent variance reduced ascension optimization algorithm for deep neural networks. In: Iyer, B., Rajurkar, A., Gudivada, V. (eds) Applied Computer Vision and Image Processing. Advances in Intelligent Systems and Computing, 1155 (2020). Springer, Singapore. https://doi.org/10.1007/978-981-15-4029-5_38

32. Kanayama, Y., Kimura, Y., Miyazaki, F., Noguchi, T.:A stable tracking control method for an autonomous mobile robot. In: IEEE International Conference on Robotics and Automation, 1, pp. 384-389 (1990). https://doi.org/10.1109/ROBOT.1990.126006

33. Zhu, X., Dong, G., Hu, D., Cai, Z.:Robust stabilization of wheeled mobile robots moving on uncertain uneven surface. In: Sixth International Conference on Intelligent Systems Design and Applications, pp. 126-131 (2006). https://doi.org/10.1109/ISDA.2006.43

34. Zhai, J., Song, Z.:Adaptive sliding mode trajectory tracking control for wheeled mobile robots. International Journal of Control. 92(10), 2255-2262 (2019). https://doi.org/10.1080/00207179.2018.1436194 


\section{Declarations}

We would like to submit the enclosed manuscript entitled "A model-based parameter optimization control strategy for trajectory tracking with torque and velocity constraints" to be considered for publication in "Nonlinear Dynamics". No conflict of interest exists in the submission of this manuscript, and the manuscript is approved by all authors for publication. I would like to declare on behalf of my co-authors that the work described was original research that has not been published previously, and is not under consideration for publication elsewhere, in whole or in part. All the authors listed have approved the manuscript that is enclosed. 


\section{Data Availability Statements}

The datasets generated during and/or analysed during the current study are available from the corresponding author on reasonable request.

The data that support the findings of this study are not openly available due to the follow-up related research. As there are many relevant experimental data, we can provide them according to demand. 